\title{
Erratum
}

\section{Erratum to: On a Certain Approach to Quantum Homogeneous Spaces}

\author{
P. Kasprzak \\ Department of Mathematical Methods in Physics, Faculty of Physics, Warsaw University, Warsaw, Poland. \\ E-mail: pawel.kasprzak@fuw.edu.pl
}

Received: 29 October 2014 / Accepted: 8 December 2014

Published online: 13 February 2015 - C Springer-Verlag Berlin Heidelberg 2015

Commun. Math. Phys. 313, 237-255 (2012)

Definition 0.1 Let X be a locally compact space with a transitive action of G. We say that $\mathrm{X}$ is a homogeneous $\mathrm{G}$-space if it can be identified with $\mathrm{G} / \mathrm{H}$, i.e., there exists a homeomorphism $\Phi: X \rightarrow \mathrm{G} / \mathrm{H}$ such that $\Phi(g x)=g \Phi(x)$ for all $g \in \mathrm{G}$ and $x \in \mathrm{X}$.

It turns out that if $\mathrm{G}$ is $\sigma$-compact, then $\mathrm{X}$ with a transitive action of $\mathrm{G}$ is automatically homogeneous - see [1] for the proof and for the examples of the G-spaces with transitive action that are not homogeneous. In the original article we made a mistake of not making a clear distinction between homogeneous G-spaces and transitive G-spaces. This led us to a gap in the proof of Theorem 5.1, which we fill in what follows. In fact, throughout the paper one should replace the sentences in which the word "transitive" appears by the appropriate version with the word "homogeneous".

Theorem 0.2 Let $\mathrm{G}$ be a locally compact group and $\mathbb{G}=\left(\mathrm{L}^{\infty}(\mathbb{G}), \Delta_{\mathbb{G}}\right)$ the corresponding LCQG. Let $\left(N, \Delta_{N}\right)$ be a quantum homogeneous space in the sense of Definition 3.1 in the original article with $N$ being commutative. Then the spectrum $\mathrm{X}=\mathrm{Sp}(\mathbb{D})$ is a homogeneous $\mathrm{G}$-space and $\left(N, \Delta_{N}\right)=\left(\mathrm{L}^{\infty}(\mathrm{X}), \Delta_{\mathrm{X}}\right)$.

Proof. Let us fix a point $\tau \in \mathrm{X}$ and $\operatorname{let}_{\mathrm{ev}}: \mathrm{C}_{0}(\mathbb{D}) \rightarrow \mathbb{C}$ be the corresponding character. Let us consider the morphism $\pi_{\mathbb{D}} \in \operatorname{Mor}_{\mathbb{G}}(\mathbb{D}, \mathbb{G})$ given by the formula $\pi_{\mathbb{D}}(d)=(\iota \otimes$ $\left.\mathrm{ev}_{\tau}\right) \Delta_{\mathbb{D}}(d)$ for any $d \in \mathrm{C}_{0}(\mathbb{D})$. Using $\mathbb{G}$-simplicity of $\mathbb{D}$ (see Theorem 4.2 in the original article) we get the injectivity of $\pi_{\mathbb{D}}$.

In what follows, we shall show that $\pi_{\mathbb{D}}$ may be extended to an injective normal *-homomorphism of $\pi_{N}: N \rightarrow \mathrm{L}^{\infty}(\mathbb{G})$. The strictness of $\Delta_{N}$ gives $\Delta_{N}(N) \subset$ $\mathrm{M}\left(\mathcal{K}\left(\mathrm{L}^{2}(\mathbb{G})\right) \otimes \mathrm{C}_{0}(\mathbb{D})\right)$, which enables us to define $\pi_{N}(x)=\left(\iota \otimes \mathrm{ev}_{\tau}\right) \Delta_{N}(x)$ for 
any $x \in N$. Let $I=\operatorname{ker} \pi_{N}$ and let $p \in N$ be the central projection generating $I$ : $I=p N$. Using the equality

$$
\left(\iota \otimes \pi_{N}\right) \circ \Delta_{N}=\Delta_{\mathbb{G}} \circ \pi_{N}
$$

we may see that $\Delta_{N}(I) \subset \mathrm{L}^{\infty}(\mathbb{G}) \bar{\otimes} I$. In particular $\Delta_{N}(p) \leq 1 \otimes p$. Proceeding as in the proof of Theorem 4.2 in the original article we get the equality $\Delta_{N}(p)=1 \otimes p$. The ergodicity of $\Delta_{N}$ implies that either $p=0$ or $p=1$. The case $p=1$ is ruled out by the injectivity of $\pi_{\mathbb{D}}$ hence $p=0$ and $\pi_{N}$ is injective. Identifying $N$ with its image in $\mathrm{L}^{\infty}(\mathbb{G})$ under $\pi_{N}$ end using (1) we get $\Delta_{N}=\left.\Delta_{\mathbb{G}}\right|_{N}$, thus $N \subset \mathrm{L}^{\infty}(\mathbb{G})$ is a coideal: $\Delta_{\mathbb{G}}(N) \subset \mathrm{L}^{\infty}(\mathbb{G}) \bar{\otimes} N$. Using [2, Theorem 2] we get a closed subgroup $\mathrm{H} \subset \mathrm{G}$ such that $N=\mathrm{L}^{\infty}(\mathrm{G} / \mathrm{H})$. Thus [3, Theorem 6.1] enables us to identify $\mathrm{Sp}(\mathbb{D})$ with $\mathrm{G} / \mathrm{H}$.

\section{References}

1. Blaavand, J.L.: Homogeneous spaces Internet notes. http://home.imf.au.dk/blaavand/unitary.pdf

2. Takesaki, M., Tatsuuma, N.: Duality and subgroups. Ann. Math. 93, 344-364 (1971)

3. Vaes, S.: A new approach to induction and imprimitivity results. J. Funct. Anal. 229(2), 317-374 (2005)

Communicated by Y. Kawahigashi 